\title{
Image synthesis M/2D/HWT in VLSI Technology
}

\author{
P. Maniraj Kumar, P. Thirumurugan, P.Karthikeyan, S. Saravanan, J. Kumarnath
}

\begin{abstract}
Image synthesis is grouping of valid information from a group of images in to unique image. The ensuing image is an improved quality than any other images. The spectral deformation major con in standard method. The different multiscale transforms are proposed the overcome the issue. The image is affected by impulse noise because of satellite images, are filtered the impulse noise in the image syntehesis. In this paper we studied the architecture of Edge preserve algorithm which good in PSNR and MSE. The proposed technique using wavelet decomposition is implemented in Matlab for low resolution images are multispectral image and high resolution image are panchromatic image and then we combined the synthesized image in Altera Cyclone. The result shows the significant area and power. The synthesis image has a colour combination which is effective than any other in set of images.
\end{abstract}

KEYWORDS: Image Fusion, Haar Wavelet, Edge preserving Filter, Impulse Noise.

\section{INTRODUCTION}

Data are taken from various sources are merged and then they are organized to make optimal decisions. [1].The satellite images are captured which have the characteristic of various spectral, temporal, spatial resolution in various band of frequency In the field of remote sensing, the data acquired from all these sources have a disparity in nature and images of same locations have spatial dependency. This situation is exploited by image fusion algorithms to come up with merged images which are more detailed and information-rich than any of the individual images [2].

The covered area, researcher's knowledge and quality of image will reflect on the degree of accuracy, hence the application varies from border security to growth detection in agro-products. However, it will not suit for any application if the data is distorted largely and low pixel resolution. Unfortunately the distortion and resolution in real world are more severe than the hardware designed for specific application [3]; fusion plays a very important role in such cases. It is able to convert to higher information content with the low information data sets.

\subsection{Background}

The combination of panchromatic image (i.e. High resolution image) and Multispectral image (i.e. low resolution image) is widely used techniques for image

Revised Manuscript Received on October 15, 2019.

Dr. P. Maniraj Kumar, Professor, PSNA College of Engineering and Technology,Dindigul, Tamil Nadu, India.

(Email::mani1376@yahoo.com)

Dr. P. Thirumurugan, Associate Professor, PSNA College of Engineering and Technology, Dindigul, Tamil Nadu, India

Dr. P. Karthikeyan, Associate Professor, PSNA College of Engineering and Technology, Dindigul, Tamil Nadu, India

Mr. S. Saravanan, Associate Professor, PSNA College of Engineering and Technology, Dindigul, Tamil Nadu, India

Mr. J. Kumarnath, Associate Professor, PSNA College of Engineering and Technology, Dindigul, Tamil Nadu, India. fusion [4] \& [5]. Many image fusion methods for the purpose have been proposed but the research of interest is to generate the noise free fused image for real time implementation. Now, the growing needs in the society has leaded the way for fusing the image in real time. The immediate idea blinking here it was Field Programmable Gate Array (FPGA). Since the future enhancement of this product provides a number of pros such as, it support large number of logic elements to implement complex algorithm and it provides remarkable improvement in terms of speed, area and power.

\subsection{METHODS}

Image synthesis tcan enhance a digital image without spoil it. The spatial domain and frequency domain are the two types of image enhancement method.The image is first transferred in to frequency domain in frequency domain methods; it means that, we have to compute the transformed image using Fourier Transform. The resultant image is obtained by performing the fusion operation in the transformed image and then we have to calculate the inverse transform, the feature in the image like image brightness, gray level distribution and contrast adjustment can be performed using enhancement operations. The pixel value of the output can be altered to the transformation function applied on the input values [6]. Pyramid based image fusion methods and Discrete Wavelet Transform (DWT) based fusion as shown in below Fig. 1 basic architecture .

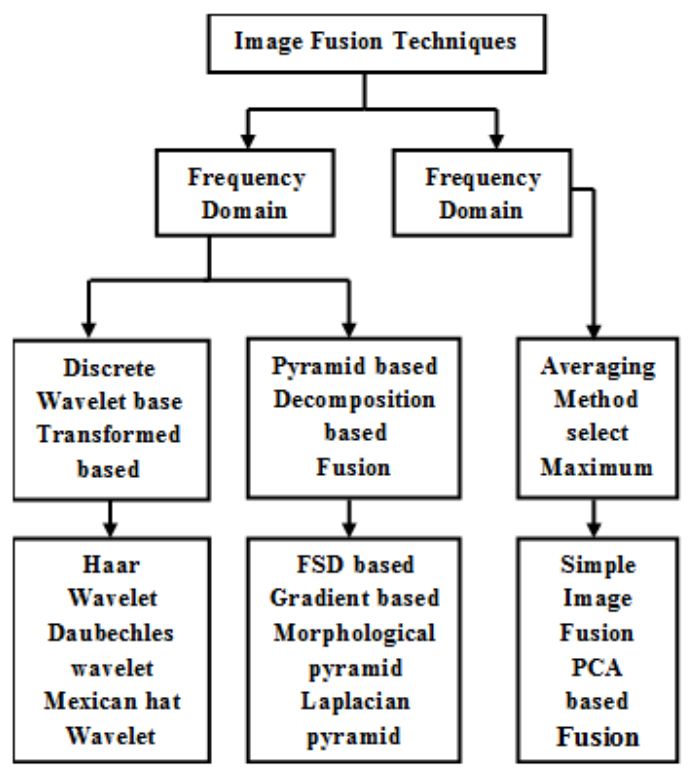

Fig. 1 Image Fusion Techniques

Published By:

Blue Eyes Intelligence Engineering

\& Sciences Publication 


\section{IMAGE FUSION USING WAVELET}

The wavelet is used for much substantive reason. Based on the application the reason may vary. For example, the time and space component of a signal can be computed using wavelet transforms and it will contribute less. This feature of wavelets is very useful in applications such as noise removal, edge detection and data compression. Generally, wavelets are beneficial when used to obtain further information from that signal that is not readily available in the raw signal [7] \& [8]. The transform of a signal is just another way of representing the signal. The information content in the signal is not altered. The transient signal is analyze to greater extent by using localized wave called as wavelet, where its concentrated energy is found in space and time. The wavelet transform has the ability to decompose complex information and patterns into elementary forms.

The track record is good in case of wavelet. They have been successfully used in many other image processing applications. The lossless reconstruction in this process is complete.

The standard methods (HIS or PCA) with wavelet transforms produce superior results [10] than either standard methods or simple wavelet-based methods alone. Her we are using Discrete Wavelet Transform for fusion process since DWT is simplest transform among numerous multiscale transform method.

\section{DISCRETE WAVELET TRANSFORM}

One dimension Discrete Wavelet Transform can be decaying again using the One dimension Discrete Wavelet Transform. This is called multi-level One dimension Discrete Wavelet Transform, the below Fig.2 shows the 2level One dimension Discrete Wavelet Transform

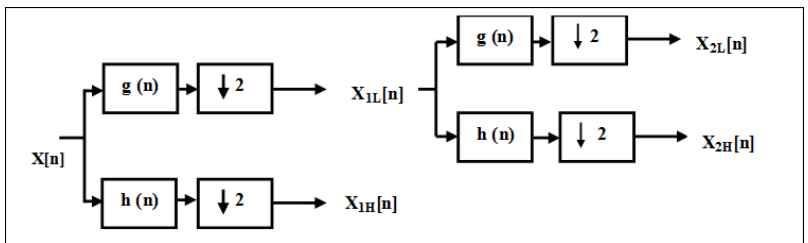

Fig. 2 The Block Diagram 2-level 1-D DWT

\subsection{Inverse discrete wavelet transforms (IDWT)}

The renovation progression from the DWT coefficients is shown in the right part of Fig.3 called inverse DWT (IDWT). The filters $h[n], g[n], h 1[n]$ and $g 1[n]$ in the figure can be designed withquadrature mirror filter (QMF) method and also Orthonormal filter method.

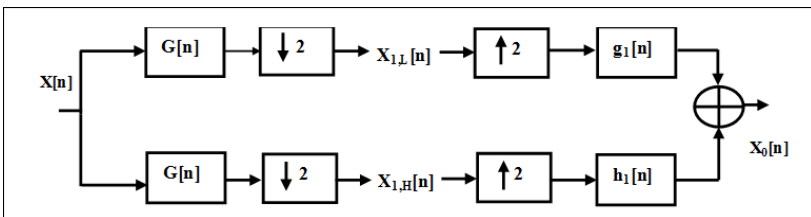

Fig. 3 The Block Diagram 2-level 1-D IDWT

\subsection{Haar Wavelet (HWT)}

The Haar wavelet is discontinuous in nature and its function will resemble as step function.

The function with HWT is defined as:

$$
\left(a^{\mathrm{L}} \mid d^{\mathrm{L}}\right) \quad \mathrm{f}
$$

$$
\begin{aligned}
& \mathrm{a}_{1}, \mathrm{a} 2, \ldots, \mathrm{a}_{\mathrm{N} / 2}=\mathrm{a}^{\mathrm{L}} \\
& \mathrm{d}_{1}, \mathrm{~d}_{2}, \ldots, \mathrm{d}_{\mathrm{N} / 2}=\mathrm{a}^{\mathrm{L}}
\end{aligned}
$$

Where decomposition level is L, approximation sub band is $\mathbf{a}$ and $\mathbf{d}$ is the detail sub band.

$$
\begin{array}{ll}
a_{m}=\frac{f 2 m+f 2 m-1}{\sqrt{2}} & \text { f or } m=1,2, \ldots, N / 2 \\
d_{m}=\frac{f 2 m-f 2 m-1}{\sqrt{2}} & \text { f or } m=1,2, \ldots, N / 2
\end{array}
$$

For example, if $\mathrm{f}=\left\{\mathrm{f}_{1}, \mathrm{f}_{2}, \mathrm{f}_{3}, \mathrm{f}_{4}, \mathrm{f}_{5}, \mathrm{f}_{6}, \mathrm{f}_{7}, \mathrm{f}_{8}\right\}$ is a time signal of length the HWT decomposes $\mathbf{f}$ into an approximation sub band containing the Low frequencies and a detail sub band containing the high frequencies:

$$
\begin{aligned}
& \text { Low }=\mathrm{a}=\{\mathrm{f} 2+\mathrm{f} 1, \mathrm{f} 4+\mathrm{f} 3, \mathrm{f} 6+\mathrm{f} 5, \mathrm{f} 8+\mathrm{f} 7\} / \sqrt{2} \\
& \text { High }=\mathrm{d}=\{\mathrm{f} 2-\mathrm{f} 1, \mathrm{f} 4-\mathrm{f} 3, \mathrm{f} 6-\mathrm{f} 5, \mathrm{f} 8-\mathrm{f} 7\} / \sqrt{2}
\end{aligned}
$$

To apply HWT on images, we first apply a one level Haar wavelet to each row and secondly to each column of the resulting "image" of the first operation. The sub bands which are multiple in numbers: LL, HL, and $\mathrm{HH}$ can be formed by decomposing the resulted image. (Low as L, High as H). The LL-sub band contains an approximation of the original image while the other sub bands contain the missing details. We can decompose the LL-sub band output at any stage. The level 1and 2 of HWT decomposition is shown below Fig. 4.

\begin{tabular}{|c|c|}
\hline LL & HL \\
\hline LH & HH \\
\hline
\end{tabular}

\begin{tabular}{|c|c|c|}
\hline LL & HL & \multirow{2}{*}{ HL } \\
\cline { 1 - 2 } LH & HH & \\
\hline LH & HH \\
\hline
\end{tabular}

Fig. 4 HWT Decomposition Level

\section{PROPERTIES OF HAAR WAVELET}

The following properties describe the Haar transform: a) Haar transform is orthogonal and real. Therefore $\mathrm{Hr}=\mathrm{Hr}^{*}$ $\mathrm{Hr}^{-1}=\mathrm{Hr}^{\mathrm{T}}$

Haar Transform is very fast transform.

b) The basic vector of Haar matrix is sequency ordered.

c) Energy compaction for images in Haar transform is under consideration.

d) Orthogonality: Splitting the high and low frequency part of the original signal with filter enabling and without duplicating the information is called as orthogonal.

e) Linear Phase: The magnitude response of the filter should be exactly zero outside the frequency range covered by the transform. This is energy invariant property.

f) The image reconstructed is perfect

\subsection{Procedure for Haar Transform}

Step 1: Each sample pair is taken and average should be finding ( $\mathrm{n} / 2$ average).

Step 2: The average and sample's difference is calculated ( $\mathrm{n} / 2$ differences). 
Step 3: The averages are filled in the first half of the array.

Step 4: The differences are filled in the second half of the array.

Step 5: The process in the first half of the array is repeated.

\section{PROPOSED METHOD}

\section{$5.1 M-2 D-H W T$}

The Modified 2D Haar Wavelet Transform is the proposed method for fusion process. Since it has advantages of;

1) Computation Speed is high.

2) It is more efficient in terms of area and speed during real time implementation

3) M-2D-HWT is efficient Fusion method.

4) It doesn't require temporary register hence if we implement in Altera board means the area is consumption is reduced.

5) Simplicity operation and so the verification is easy.

6) Final fused image Color is adjustable, etc.

\subsection{Proposed Algorithm}

Step 1: Read the image from different sources (sensors, $\mathrm{x}$ ray Machine, user).

Step 2: The two images should be resized for resolution.

Step 3: 2-D DWT is applied using Haar wavelet.

Step 4: The Haar transform is computed based on the different level of decomposition.

Step 5: The transform should be taken for the R, G, and B value.

Step 6: Then the transformed value of different image is added.

Step 7: The inverse transform is taken.

Step 8: Display the Final fused image.

Step 9: Calculate the PSNR for the final image.

\section{IMPLEMENTATION METHODOLOGY}

\subsection{Software}

In software implementation phase, shown in Fig.5 use Matlab tool to fuse the image from different sources. In initial step we have to calculate the $R, G, B$ value of the images from source, then each value is given to resize block for resizing and we have to take transform using M-2DHWT.Then transformed values of R, G and B of image 1 and image 2 are given to fusion block followed by inverse transform of the image and finally display the fused image.

\subsection{Hardware}

The architecture for hardware implementation of image fusion is shown in Fig 6. The image from different source is stored in ROM namely ROM1 and ROM2 then corresponding value is given to the Fusion block after computing the DWT. To remove the impulse noise in the fused image filtering process is done using edge preserving algorithm as in [11]. The Hardware architecture for the algorithm is shown in Figure as in 5(a). The algorithm [12] comprises of five main blocks namely, register bank, threshold block, line buffers (odd \& even) and mean filter. Line Buffer-odd and Line Buffer-even stores the pixel at odd and even row. The pixel to be denoised has been stored in Register Bank, threshold block is used to compare the normal pixel with noisy one and finally the filtered image is received from the mean filter and should be stored in RAM. Then the Inverse DWT is taken for the image which is stored in RAM and display via VGA port using VGA Controller.

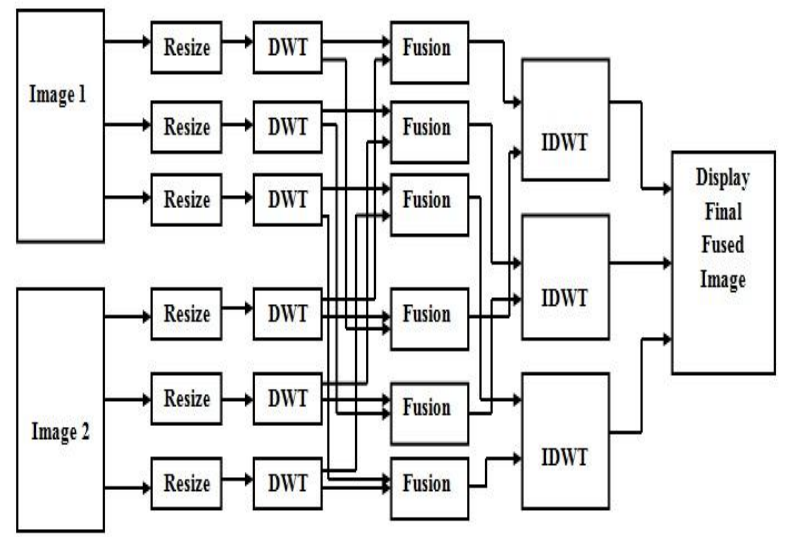

Fig. 5 Block Diagram for Software Model

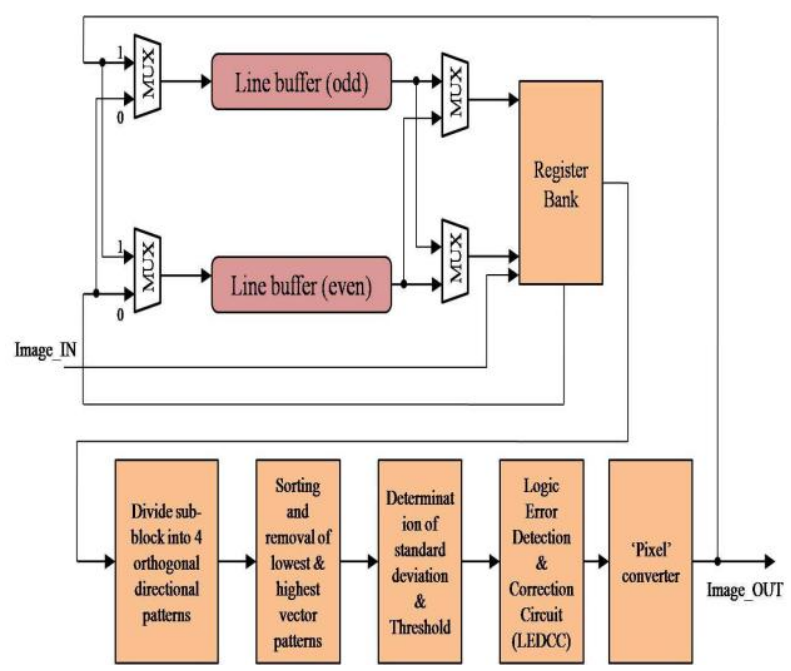

Fig. 5 (a) Edge Preserving Filter

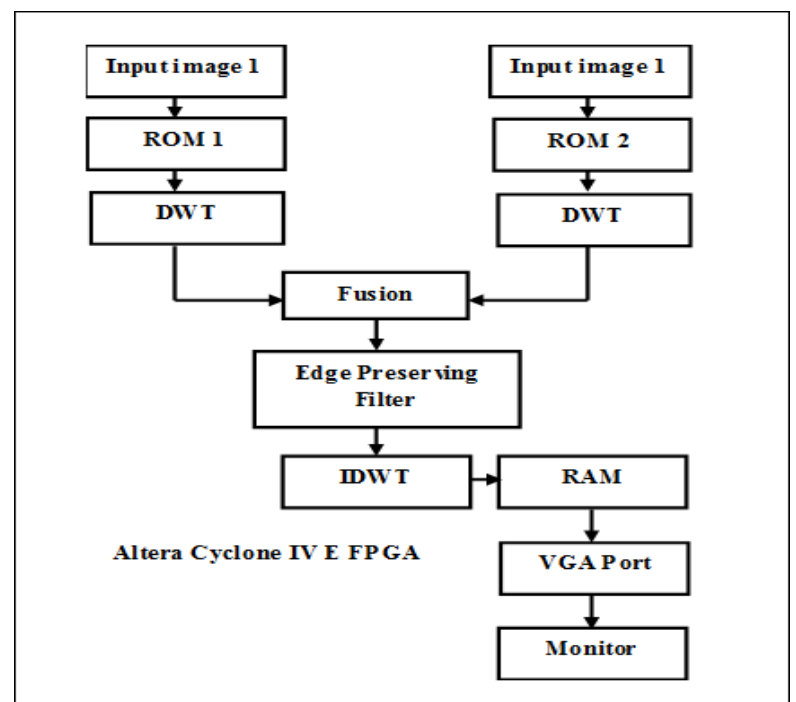

Fig. 6 Block Diagram for Hardware Model 
Table 1. Results of resources in relation to image on an ALT cyclone IV e ep4ce115f29c7 FPGA

\begin{tabular}{|ccc|}
\hline Devices & EP2C35F672 & EP4CE115F29C7 \\
Image Size & $\mathrm{N} \times \mathrm{M}$ & $\mathrm{N} \times \mathrm{M}$ \\
$\begin{array}{c}\text { Logic Register } \\
\text { (A/U) }\end{array}$ & $2828 / 34593$ & $2773 / 114480$ \\
LUT/LC & 2828 & 2767 \\
$\begin{array}{c}\text { Logic } \\
\text { Cells(A/U) }\end{array}$ & $9294 / 33216$ & $9127 / 114480$ \\
$\begin{array}{c}\text { Memory Bits } \\
(\mathrm{A} / \mathrm{U})\end{array}$ & $70 / 483840$ & $69 / 3981312$ \\
$\begin{array}{c}\text { Multiplier 9-bit } \\
(\mathrm{A} / \mathrm{U})\end{array}$ & $57 / 70$ & \\
\hline
\end{tabular}

Table 2 . Results of resources in relation to bit length on an ALT CYCLONE IV E EP4CE115F29C7 FPGA

\begin{tabular}{|c|c|c|}
\hline Devices & $\begin{array}{l}\text { CYCLONE II } \\
\text { EP2C35F672 }\end{array}$ & $\begin{array}{l}\text { CYCLONE IVE } \\
\text { EP4CE115F29C7 }\end{array}$ \\
\hline No Channels & 16 & 16 \\
\hline $\begin{array}{c}\text { Logic Register } \\
(\mathrm{A} / \mathrm{U})\end{array}$ & R828/34593 & $2773 / 114480$ \\
\hline LUT/LC & 2828 & 2767 \\
\hline$\underset{\text { Cells }(\mathrm{A} / \mathrm{U})}{\operatorname{Logic}}$ & $9294 / 33216$ & $9127 / 114480$ \\
\hline $\begin{array}{c}\text { Memory Bits } \\
\text { (A/U) }\end{array}$ & $70 / 483840$ & $69 / 3981312$ \\
\hline $\begin{array}{l}\text { Multiplier 9-bit } \\
\text { (A/U) }\end{array}$ & $57 / 70$ & $55 / 532$ \\
\hline
\end{tabular}

Table 3. SNR versus Bit Length of the Proposed System

\begin{tabular}{|ccc|}
\hline Devices & $\begin{array}{c}\text { CYCLONE II } \\
\text { EP2C35F672 }\end{array}$ & $\begin{array}{c}\text { CYCLONE IV E } \\
\text { EP4CE115F29C7 } 7\end{array}$ \\
Bit-Length & 16 & 16 \\
SNR(dB) & 98,15 & 106,77 \\
\hline
\end{tabular}

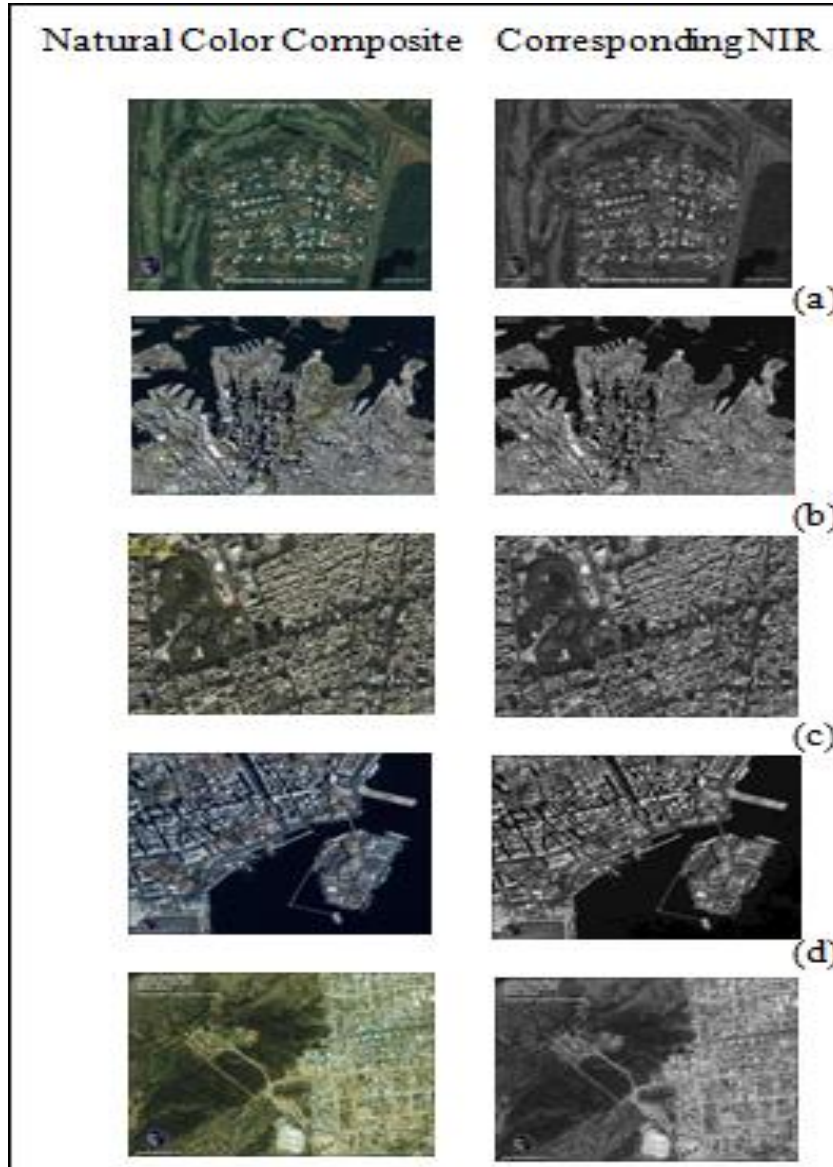

(a)

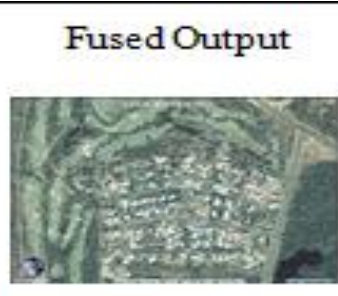

Filtered Output
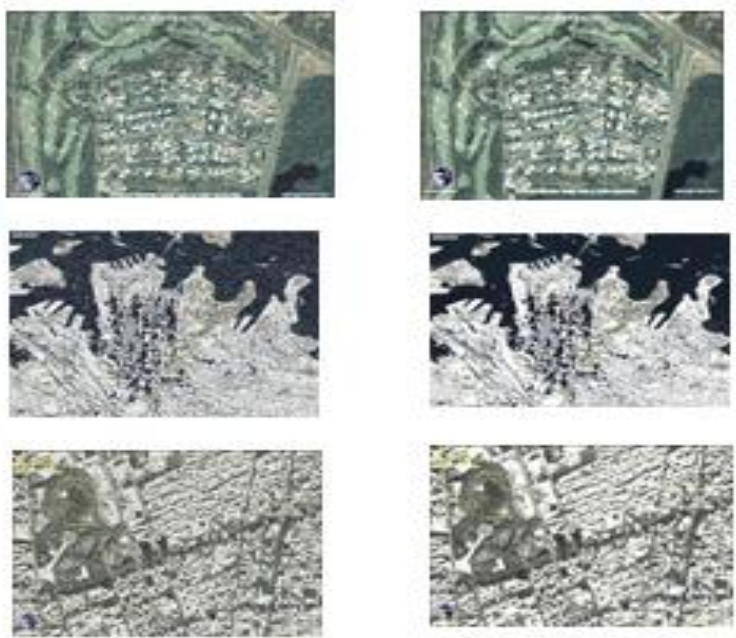

)
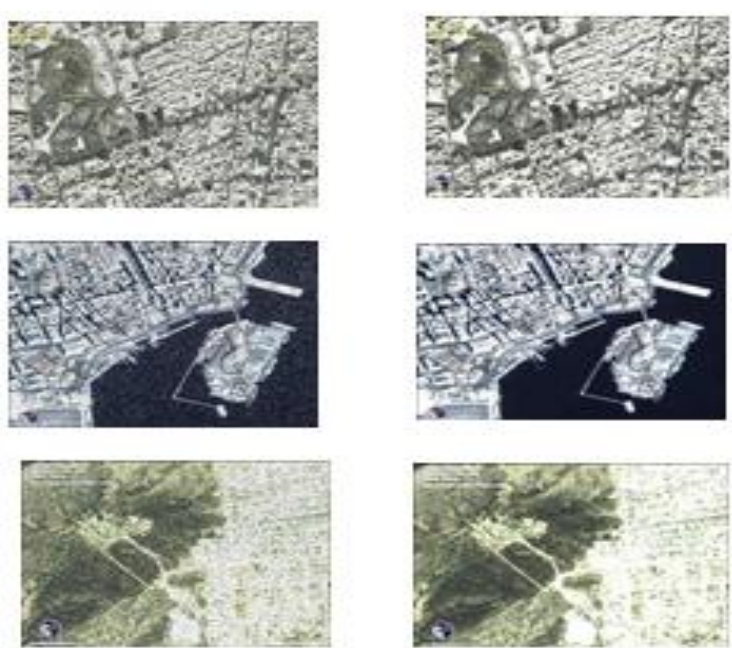
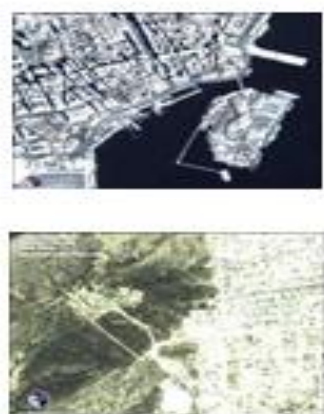

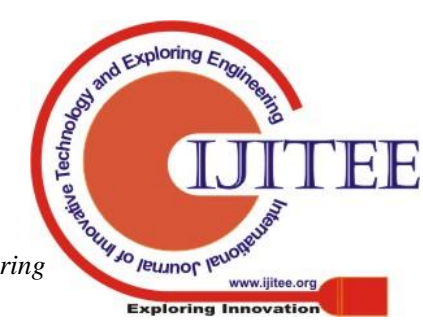




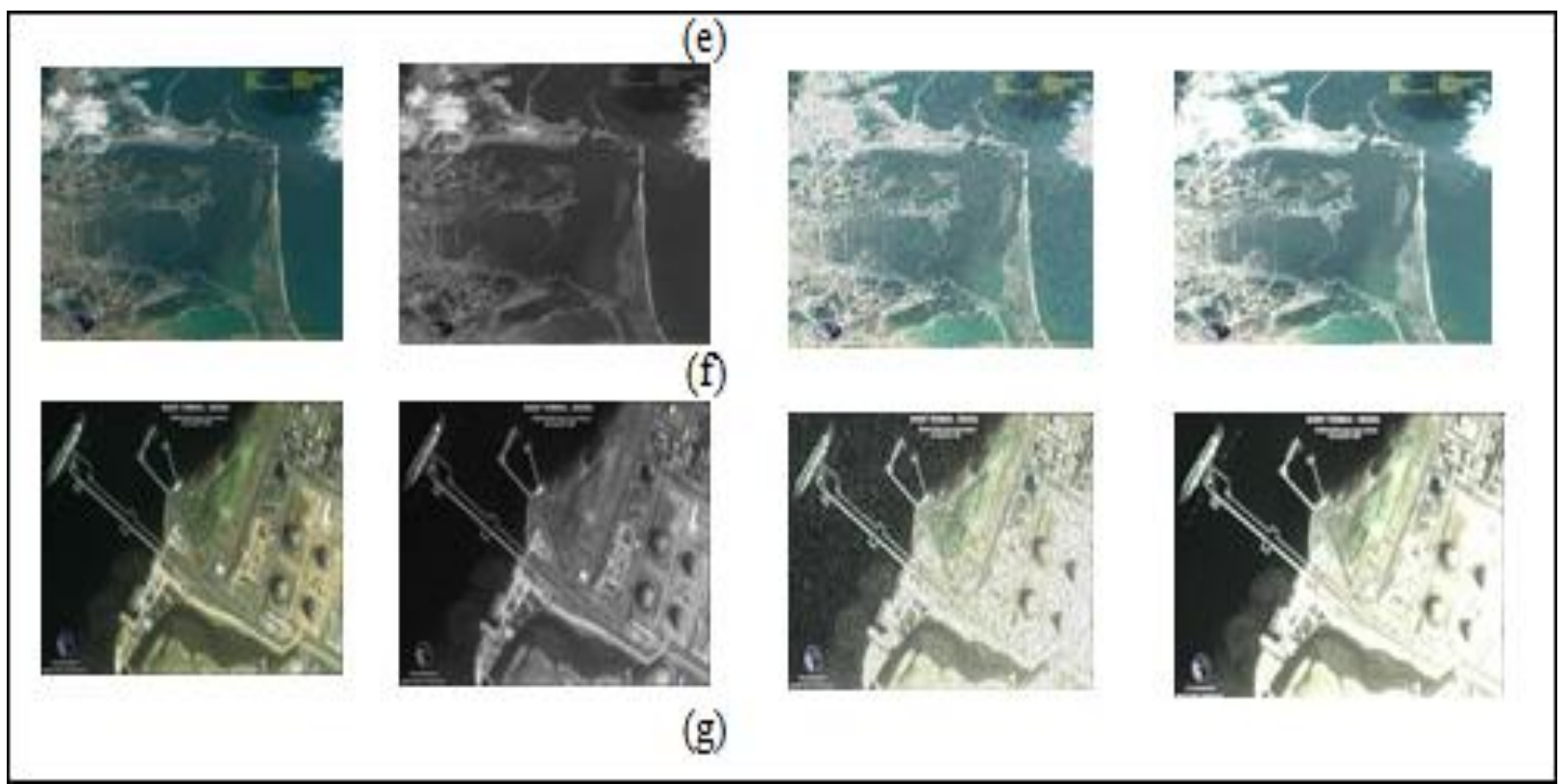

TABLE 4. Performance Compassion For The Proposed Algorithm With Reference Algorithm

\begin{tabular}{|lllllllllll|}
\hline IKONOS & \multicolumn{3}{c}{ PA } & & & \multicolumn{5}{c|}{ DWT } \\
DATA & & & & & & & & & \\
SET & PSNR & MSE & NCC & MD & NAE & PSNR & MSE & NCC & MD & NAE \\
Abuja & 39.55 & 7.26 & 1.8 & 49 & 0.91 & 38.39 & 9.49 & 1.05 & 19 & 0.05 \\
Sydney & 38.48 & 9.28 & 1.61 & 48 & 0.78 & 36.46 & 14.77 & 1.07 & 18 & 0.07 \\
Iran & 38.2 & 9.9 & 1.65 & 38 & 0.79 & 35.5 & 18.46 & 1.06 & 27 & 0.06 \\
Brazil & 39.48 & 7.37 & 1.64 & 41 & 0.9 & 37.67 & 11.19 & 1.06 & 12 & 0.07 \\
Mexico & 38.02 & 10.32 & 1.59 & 46 & 0.7 & 34.64 & 22.51 & 1.05 & 21 & 0.05 \\
Japan & 38.72 & 8.78 & 1.77 & 64 & 0.86 & 36.11 & 16.03 & 1.04 & 19 & 0.05 \\
Nigenia & 38.6 & 9.04 & 1.65 & 76 & 0.75 & 36.26 & 15.47 & 1.06 & 15 & 0.06 \\
\hline
\end{tabular}

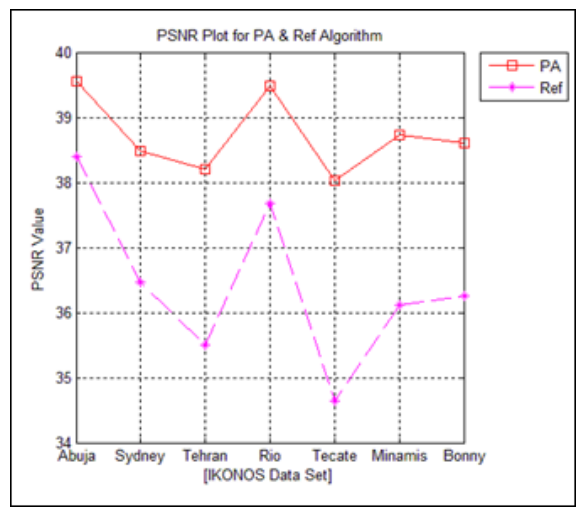

Fig. 8 PSNR plot for PA \& Reference Algorithm

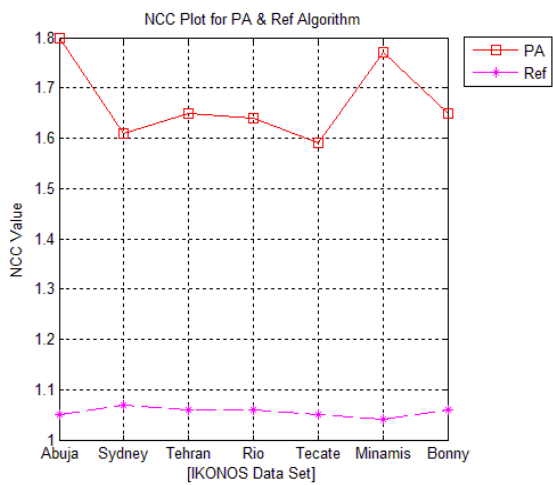

Fig 9. NCC plot for PA \& Reference Algorithm

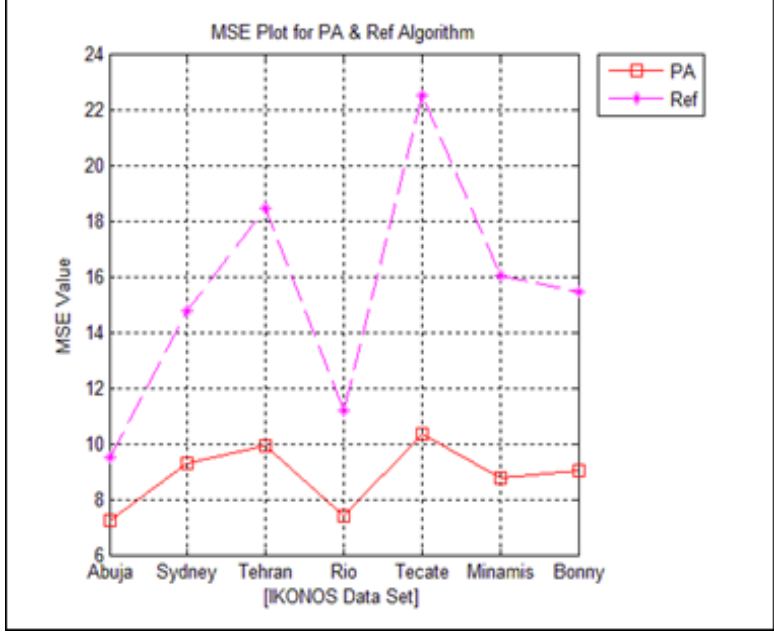

Fig. 10 MSE plot for PA \& Reference Algorithm

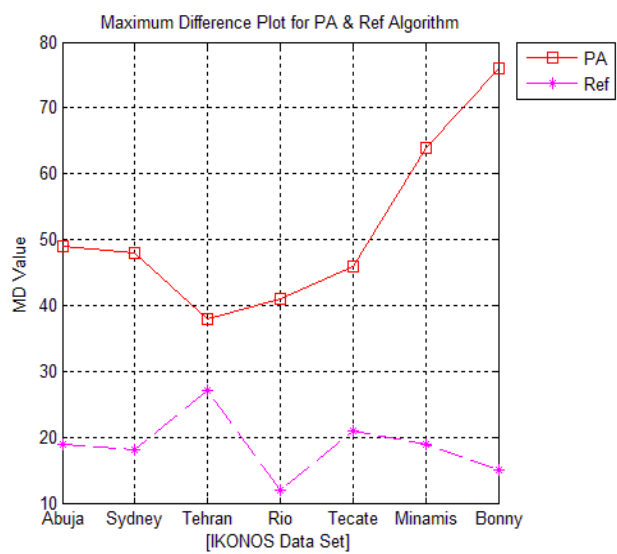

Fig. 11 MD plot for PA \& Reference Algorithm

Published By:

Blue Eyes Intelligence Engineering

\& Sciences Publication

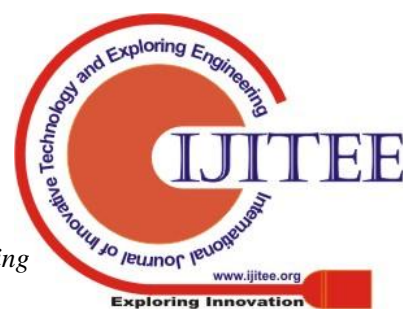




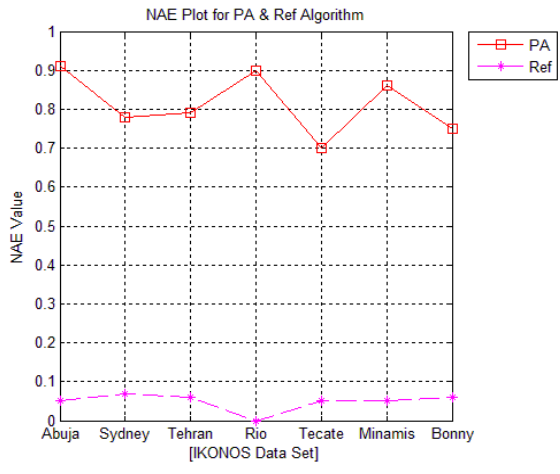

Fig. 12 NAE plot for PA \& Reference Algorithm

\section{QUALITY MEASUREMENT}

A comparison is performed with several filters and the proposed result in terms of MSE, PSNR, NCC, MD and NAE. The comparative study for the above mentioned parameters is studied and plotted in Table 4 and figure 8-12 respectively.

Where MSE - Mean Square Error

PSNR - Peak Signal to Noise Ratio

NCC -Normalized Cross Section

$$
\begin{gathered}
M S E=\frac{1}{M N} \sum_{j=1}^{M} \sum_{k=1}^{N}\left(x_{j, k}, x_{j, k}^{\prime}\right)^{2} \\
P S N R=10 \log \frac{255^{2}}{M S E} \\
N C C=\frac{\sum_{j=1}^{M} \sum_{k=1}^{N} x_{j, k}, x_{j, k}^{\prime}}{\sum_{j=1}^{M} \sum_{k=1}^{N} x_{j, k}^{2}} \\
M D=\operatorname{Max}\left(\left|x_{j, k}-x_{j, k}^{\prime}\right|\right) \\
N A E=\frac{\sum_{j=1}^{M} \sum_{k=1}^{N}\left|x_{j, k}-x_{j, k}^{\prime}\right|}{\sum_{j=1}^{M} \sum_{k=1}^{N}\left|x_{j, k}\right|}
\end{gathered}
$$

\section{EXPERIMENTAL VERIFICATION \& RESULTS}

The proposed architecture was verified using a test bench using Altera DSP and interfaced with Altera cyclone IV E EP4CE115F29C7 FPGA.

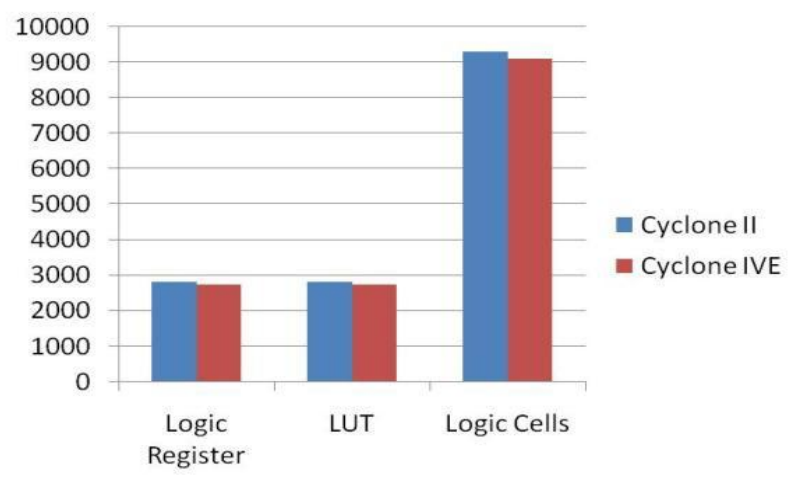

Fig. 13 Cyclone II vs. Cyclone
Table. 5 Performance measure based on PSNR and MSE

\begin{tabular}{lll}
\hline & \multicolumn{2}{l}{ Filter Output } \\
& PSNR & MSE \\
Abuja & 38.66 & 8.9023 \\
Brazil & 34.31 & 24.2704 \\
Iran & 35.88 & 16.9017 \\
Japan & 37.94 & 10.5421 \\
Mexico & 34.73 & 22.025 \\
Nigeria & 33.98 & 26.2019 \\
Sydney & 33.71 & 27.8349 \\
\hline
\end{tabular}

To verify the proposed fusion implementation, seven different data sets are employed from IKONOSdatabase (a)Abuja_Nigeria,(b) SydneyAustralia, (c)Tehran_Iran, (d)Rio_Brazil, (e)Tecate_Mexico,(f)Minamis-nrikucho_Japan,(g)Bonny Terminal_Nigeria. For the experimental analysis the Natural Color Composite of the image and its corresponding NIR channel processed image is taken for the fusion. Fused output for the data sets is shown in the Fig. 7 At the same time PSNR \& MSE calculation are done for the test images in Table4 and plotted in Fig. 8 and Fig. 9

\section{CONCLUSION}

This paper presents a fusion of images taken at different time of the same location. The M-2D-HWT algorithm performs well under the situation of spectral distortion. An added barrier that affects the efficiency of fusion image is the impulse noise produced form satellite image. In this work, impulse noise is removed to a greater extent by the use of edge preserving filter. From the result analysis under various parameters which is tabulated, we conclude that the fused image is free from noise and FPGA implementation produces remarkable results in terms of area, PSNR and MSE.

\section{REFERENCES}

1. RD 128, F-91767 Palaiseau Cedex FRANCE, "Cognitive Situation Awareness for Information Superiority", New York: Academic, 2012.

2. E. O. Brigham, "The Fast Fourier transform and its Applications", Englewood Cliffs, N.J., Prentice Hall, 2011.

3. P.Paul and P. Girija, "A Novel VLSI Architecture for Image Compression", Proc. of the 8th IEEE International Symposium on Multimedia, pp. 794-795, 2012. 
4. G. Strang and T. Nguyen, "Wavelets and Filter Banks", Wellesley Cambridge Pr, 2012.

5. ISO/IEC, JPEG Committee Draft 15444-1, 2012.

6. Hsu S. H., Gau P. W., I-Lin Wu I., and Jeng J. H., 2011,"Region-Based Image Fusion with Artificial Neural Network". World Academy of Science, Engineering and Technology, 53, pp $156-159$

7. A. Haj, "Combined DWT-DCT Digital Image Watermarking", Journal of Computer Science, Science Publication, vol. 3, no. 9, pp. 740-746, 2010.

8. Anjali Malviya1, S. G. Bhirud a short paper on „Image Fusion of Digital Imagese in International Journal of Recent Trends in Engineering, Vol 2, No. 3, November 2011 pp. 146-148.

9. Konstantinos G. Nikolakopoulos a paper on "Comparison of Nine Fusion Techniques for Very High Resolution Data" Photogrammetric Engineering \& Remote Sensing, Vol. 74, No. 5, May 2012, pp. 647-659.

10. Zhang J., 2012. "Multi-source remote sensing data fusion: status and trends", International Journal of Image and Data Fusion, Vol. 1, No. 1, pp. 5-24.

11. P.Thirumurugan and Dr. S.Sasikumar. "Performance Analysis of Impulse Noise Reduction using Pseudo Random Methodology" Medwell journals publisher on Research Journal of Applied Sciences,has been accepted for publication. ISSN: 1815932X, E ISSN: 19936079.

12. P.Thirumurugan and Dr. S.Sasikumar. FPGA implementation of Impulse noise reduction Medwell journals publisher on Research on Asian Journal of Information Technology has been initially accepted for publication. ISSN: 16823915, E ISSN: 19935994. 